\title{
Long-term follow-up of endovascular coil embolization for cerebral aneurysms using three-dimensional time-of-flight magnetic resonance angiography
}

\author{
Kentaro Hayashi*, Naoki Kitagawa*, Minoru Morikawa* ${ }^{\dagger}$, Nobutaka Horie*, \\ Junichi Kawakubo*, Takeshi Hiu*, Keisuke Tsutsumi* and Izumi Nagata*
}

Departments of *Neurosurgery and ${ }^{\dagger}$ Radiology, Nagasaki University School of Medicine, Nagasaki, Japan

Objectives: As endovascular treatment becomes more prevalent, aneurysm recurrence from neck remnants, recanalization, incomplete obliteration and bleeding remain major concerns. In the current analysis, we attempted to identify factors related to disease progression and clinical outcome in patients treated with coil embolization.

Methods: This study included 58 patients who underwent endovascular coil embolization for treatment of intracranial aneurysm. The result of embolization was evaluated with threedimensional time-of-flight magnetic resonance angiography (TOF MRA) and classified as a complete occlusion, a residual neck (minor, central and marginal types), a residual dome (central and marginal types). Patients were followed up clinically and radiologically. Statistical analyses were performed to establish factors that influenced the occurrence of adverse events such as recurrence of aneurysm.

Results: Overall, the complete occlusion rate was $18.8 \%$, the occurrence of a residual neck was $67.2 \%$, and the residual dome rate was $14.1 \%$. The mean clinical follow-up was 31.2 months. Recurrences were found in 18 aneurysms, and major recurrences were retreated with coiling or surgery. The post-treatment study revealed that the marginal-type aneurysm filling has a significant impact on outcome. Thus, perianeurysmal edema was correlated with recurrence of the aneurysm.

Conclusions: Three-dimensional TOF MRA was a sensitive tool for visualizing residual filling of embolized aneurysm and is useful for long-term follow-up of patients. [Neurol Res 2008; 000: 000-000]

Keywords: Cerebral aneurysm; endovascular therapy; embolization; MRA; recurrence

\begin{abstract}
INTRODUCTION
Endovascular treatment with detachable coils has become an established technique for patients with intracranial aneurysm and has been associated with a better outcome than surgery at 1 -year after treatment ${ }^{1,2}$. However, aneurysm may partially recur due to coil compaction or enlargement of a remnant. Consequently, all patients with coil-treated intracranial aneurysms are observed with angiography to identify aneurysm recurrence and determine the subsequent need for additional endovascular or surgical treatment ${ }^{3}$. Previous works have demonstrated that detachable coils are compatible with magnetic resonance imaging (MRI) in terms of safety and image quality, and MRI has become a realistic diagnostic option for the follow-up of intracranial aneurysm treated with coils ${ }^{4}$. Furthermore, it is reported that three-dimensional time-of-flight magnetic resonance angiography (3D TOF
\end{abstract}

Correspondence and reprint requests: Dr K. Hayashi, Department of Neurosurgery, Nagasaki University School of Medicine, 1-7-1 Sakamoto, Nagasaki 852-8501, Japan. [kenkuni@net.nagasaki-u.ac.jp] Accepted for publication June 2008.
MRA) targeted to coiled aneurysm was more sensitive to residual flow ${ }^{5,6}$.

The aim of this work was to classify the residual filling of the aneurysm treated with detachable coil and perform a follow-up using 3D TOF MRA to assess its usefulness for the detection of aneurysm recanalization. Thus, additional MRI findings such as perianeurysmal edema were evaluated. Residual dome or filling of the marginal part of the neck was correlated with an adverse event such as recanalization. Three-dimensional TOF MRA is feasible and useful in the follow-up of patients with intracranial aneurysms treated with coil placement.

\section{MATERIALS AND METHODS}

Patient demographics

From April 1999 to December 2005, 58 patients (50 women and eight men; median age, 61.2 years; range, 39-78) harboring 64 aneurysms treated by endovascular coiling underwent simultaneous angiography and MRI at follow-up. These patients were followedup clinically and radiologically at our institute for 
$>12$ months. Among the 58 patients, seven were admitted with a subarachnoid hemorrhage confirmed by a computed tomographic scan of the brain. The locations of the aneurysms were as follows: internal carotid artery $(n=42)$, basilar tip $(n=10)$, posterior inferior cerebellar artery $(n=4)$, superior cerebellar artery $(n=3)$, anterior communicating artery $(n=3)$, middle cerebral artery $(n=1)$ and posterior cerebral artery $(n=1)$. Patient selection for coil embolization was determined by a multidisciplinary fashion. The procedure with the highest possibility of success and the least risk to the patient was recommended.

\section{Coil embolization procedure}

The coil embolization procedures were performed with general anesthesia for the ruptured aneurysms and local anesthesia for the unruptured aneurysms. All procedures were performed on a biplane C-arm angiographic system without 3D reconstruction. This work did not include cases using the stent-assisted technique but included cases using the balloon remodeling technique. Access was achieved via the right femoral artery in most cases. A 6-French guide catheter was placed in the internal carotid artery or vertebral artery, depending on the aneurysm location. After placing the guide catheter, the patient was systemically heparinized, and activated clotting times were checked hourly. Using a coaxial technique, the microcatheter was navigated into the aneurysm over a microguidewire. The first coil was generally $1 \mathrm{~mm}$ smaller than the largest diameter of the aneurysm. After successful activation of a coil, an angiographic image was obtained to check coil placement. The coil was then detached and additional coils in decreasing diameters and lengths were used to obliterate the aneurysm. This process was continued until the aneurysm was completely obliterated or it was physically impossible to insert more coils.

\section{Three-dimensional TOF MRA}

MRI was performed with a 1.5-T whole-body magnetic resonance unit (Signa Horizon; General Electric Medical Systems, Milwaukee, WI, USA) with a standard head coil. A three-dimensional TOF MRA image was obtained with the parameters of 28/ 3.2 milliseconds (repetition time/echo time), flip angle of $20^{\circ}$, and one signal acquired. Ramp pulse, multislab, zero-fill interpolation techniques were also used. The field of view was $160 \mathrm{~mm}$, with a matrix of $256 \times 256$ (160) and a section thickness of $0.7 \mathrm{~mm}$ after zero-fill interpolation. The other imaging parameters included three slabs. The thickness of all slabs was $67 \mathrm{~mm}$. The thickness of one slab was $34 \mathrm{~mm}$. One slab and six overlapping slices provided a total of 96 sections. The total acquisition time was 5 minutes 33 seconds. Basal 3D TOF MRA was processed with maximum intensity projection (MIP) and multiplanar reconstruction (MPR) images closely targeted to a coiled aneurysm. In the best MPR projection, in which residual flow was most widely visualized, the morphology of the filling was evaluated.

\section{Image analysis}

Anatomic results were evaluated in a very stringent fashion and classified as previously published ${ }^{7-9}$. In brief, a complete occlusion result meant complete obliteration of the aneurysm including the neck. A residual neck was defined as the persistence of any portion of the original defect of the arterial wall as seen on any single projection but without opacification of the aneurysmal sac. Any opacification of the sac was classified as a residual dome. Furthermore, residual filling was subclassfied into a central or marginal type. Cases with slight neck remnant were considered as minor type (Figure 1). Assessments were made by an independent observer in a blinded fashion.

\section{Strategy of follow-up}

We scheduled a 3-month follow-up MRI including 3D TOF MRA. The cerebral angiograms were obtained between 6 and 12 months after embolization. If a follow-up angiogram demonstrated no evidence of recanalization, we usually schedule a 6-month followup MRI. Results of the additional follow-up imaging studies were compared with previous images in equivalent projection. A stable remnant was defined as cases that demonstrated no change in size from its appearance on previous imaging. When aneurysmal recanalization was suspected in noninvasive studies, conventional angiography was performed immediately to check the exact state of the aneurysm and decide the necessity of further treatment. The recurrence represented any aneurysm remnant that had an increase in size or filling and was qualified as major if retreatment could theoretically be performed. Thus, new neurological episodes were noted.

\section{Statistical analysis}

The factors associated with a recurrence were determined using univariate and multivariate Cox regression analyses. Differences were considered significant if the probability value was $<0.05$.

\section{RESULTS}

\section{Clinical outcome}

At a mean follow-up period of 31.2 months, the results can be summarized as follows. One patient $(1.7 \%)$ experienced a bleeding episode. This event that occurred 4 months after treatment was associated with recurrence of the treated lesion. In this case, endovascular embolization was not suitable because the ruptured large basilar bifurcation aneurysm was accompanied with partial thrombosis. However, the patient's clinical condition was too poor to perform an open surgery; thus, coiling had been done. A newer neurological deficit occurred in one patient $(1.7 \%)$. It was associated with the mass effect of coiled aneurysm 

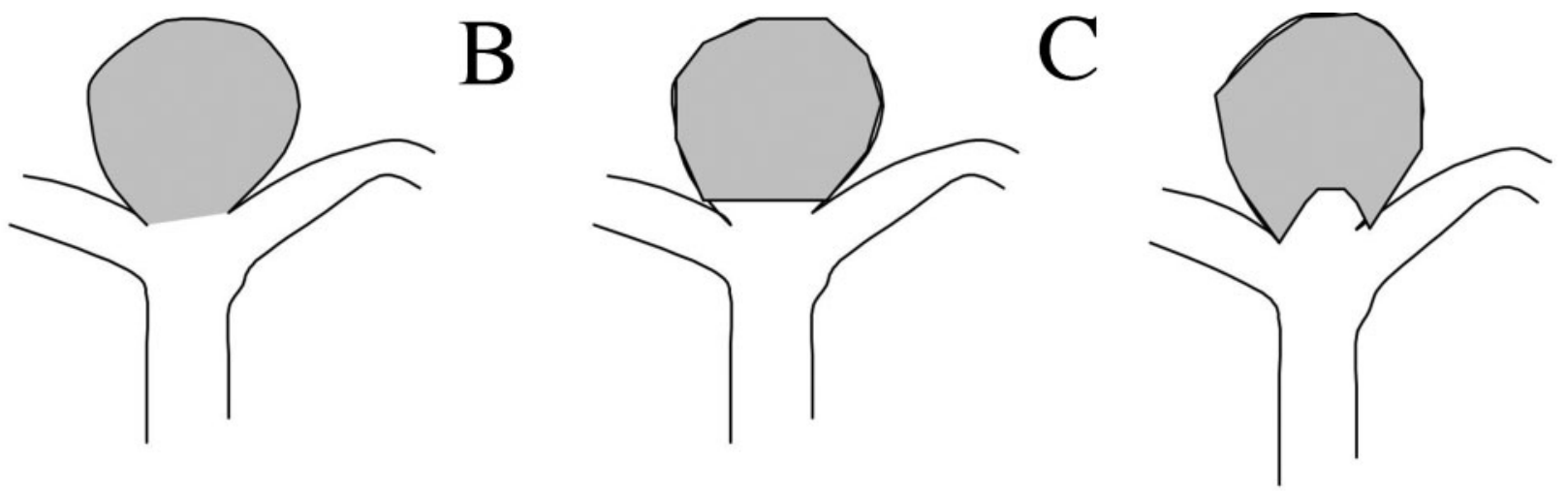

D
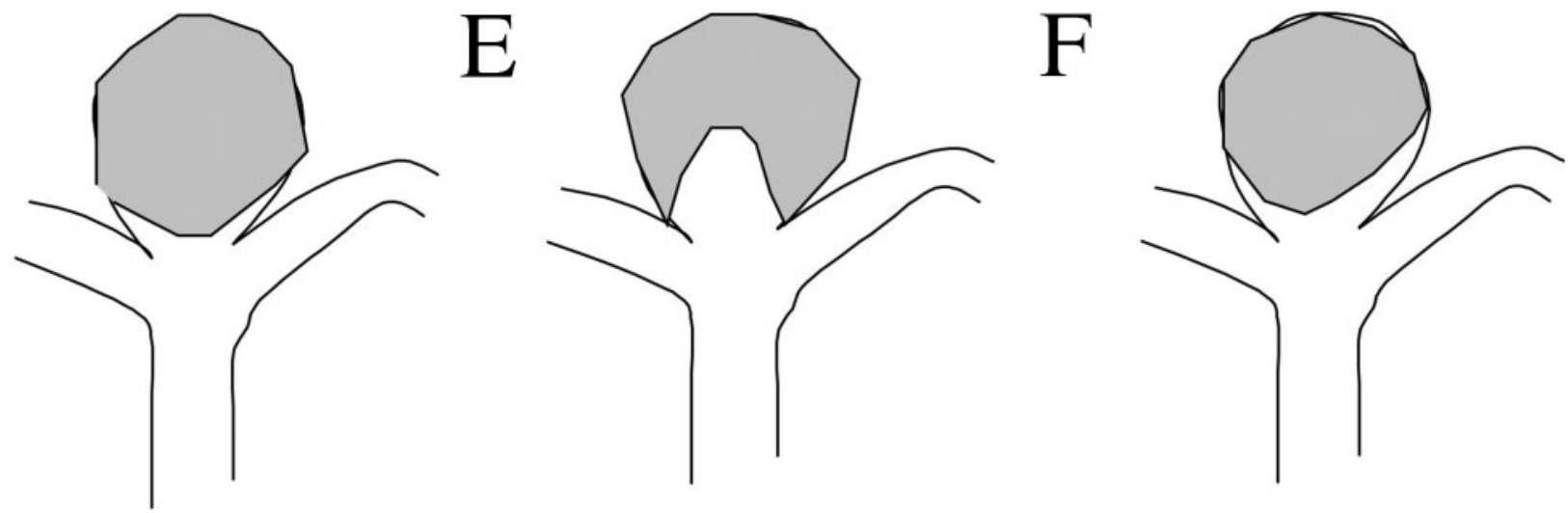

Figure 1: Classification of coil embolization: (A) complete occlusion; (B) residual neck, minor type; (C) residual neck, central type; (D) residual neck, marginal type; $(\mathbf{E})$ residual dome, central type; and (F) residual dome, marginal type

according to the recurrence. The condition of the patient improved after retreatment.

\section{Post-treatment Radiological Results}

After critical analysis of the post-treatment radiological results in multiple projections, only $16 \%(12 / 64)$ were classified as a complete occlusion, that is, no evidence of a neck remnant. Fifty-seven percent (43/64) were considered a residual neck, near-complete obliteration with a small neck remnant. Twenty-seven percent $(9 / 64)$ of the post-treatment radiological results demonstrated filling in the aneurysm dome. Of the 43 aneurysms with a residual neck, 31 were classified as minor type, seven were central type, and five were marginal type. Among those with a residual dome, six were classified as central type and three were marginal type (Table 1$)$.

\section{Radiological outcome}

Approximately $70 \%$ of the aneurysms treated with the insertion of the coils demonstrated stable results at a mean follow-up of 31.2 months. Recurrences were found in a total of $18(28.1 \%)$ of the treated aneurysms, comprising eight classified as minor and ten (15.1\%) classified as major. Among the ten major recurrences, nine were treated by endovascular coiling and one was treated by neurosurgical treatment. The post-treatment study results (residual dome) proved to have a significant impact on radiological outcome. No recanalization occurred in the completely embolized aneurysm.
Thus, the marginal-type filling was strongly correlated with the recurrence. Perianeurysmal edema was found in five cases, and all of them recurred. One of them was symptomatic due to the mass effect or focal inflammation. Moreover, outcome of the coil embolization was divided into three groups (stable coiling, minor recurrence and retreatment), and the initial size of the aneurysm was retrospectively reviewed in each group. The size of the retreatment group was significantly larger than the stable coiling group (Figure 2).

\section{Illustrative cases}

\section{Case 1}

A 50-year-old woman was admitted to our hospital because of subarachnoid hemorrhage. An angiogram

Table 1: Ratio of recurrence and retreatment

\begin{tabular}{lccc}
\hline & & Recurrence, $\mathrm{n}(\%)$ & Retreatment, $\mathrm{n}(\%)$ \\
\hline Total & 64 & $18(28.1)$ & $10(15.6)$ \\
Complete & 12 & $0(0)$ & $0(0)$ \\
Neck remnant & 43 & $12(27.9)$ & $7(16.3)$ \\
$\quad$ Minor & 31 & $8(25.8)$ & $5(16.1)$ \\
Central & 7 & $1(14.3)$ & $1(14.3)$ \\
$\quad$ Marginal & 5 & $3(60.0)$ & $2(40.0)$ \\
Residual aneurysm & 9 & $6(66.7)$ & $3(33.3)$ \\
$\quad$ Central & 6 & $3(50.0)$ & $1(16.7)$ \\
$\quad$ Marginal & 3 & $3(100)$ & $2(66.7)$ \\
\hline
\end{tabular}




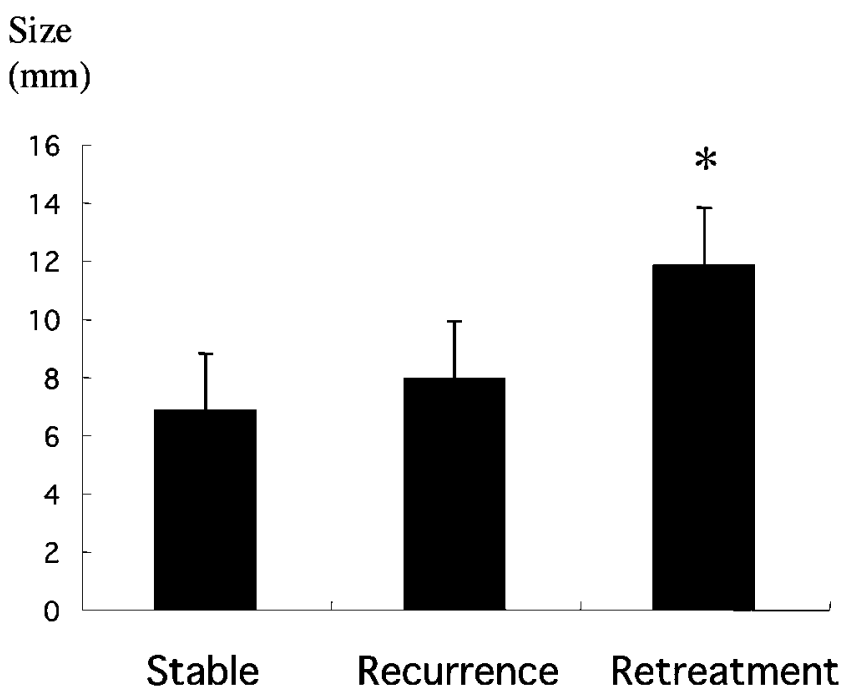

Figure 2: Initial size of the aneurysm and recurrence (stable coiling, minor recurrence and retreatment). Initial size of aneurysm retrospectively reviewed in each group. Size of retreatment group significantly larger than stable coiling group. ${ }^{*} p<0.05$ denotes significant difference showed right posterior cerebral artery aneurysm (Figure 3). The coil embolization was employed and resulted in the complete occlusion of the aneurysm. An MPR image from basal 3D TOF MRA also showed a complete occlusion of the aneurysm. The coil embolization remained unchanged 48 months after embolization.

\section{Case 2}

A 50-year-old woman with a right internal carotid artery aneurysm was treated with coil embolization. A residual neck was seen in the post-procedural control angiogram (Figure 4). An MPR image from basal 3D TOF MRA revealed residual filling inside the aneurysm, classified as a central-type residual dome. Although an MIP image of the 3D TOF MRA also showed the filling, the resolution was not sufficient. Although minor recurrence was seen 12 months later, the patient was considered protected from rebleeding. The recurrence was stable 48 months later.

\section{Case 3}

A72-year-old woman was admitted to our hospital because of incidental right internal carotid artery aneurysm (Figure 5). The result of the coiling was

\section{A}

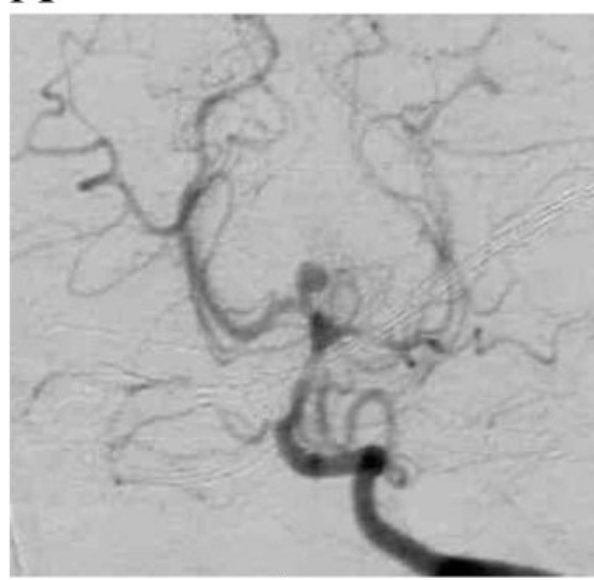

B

D

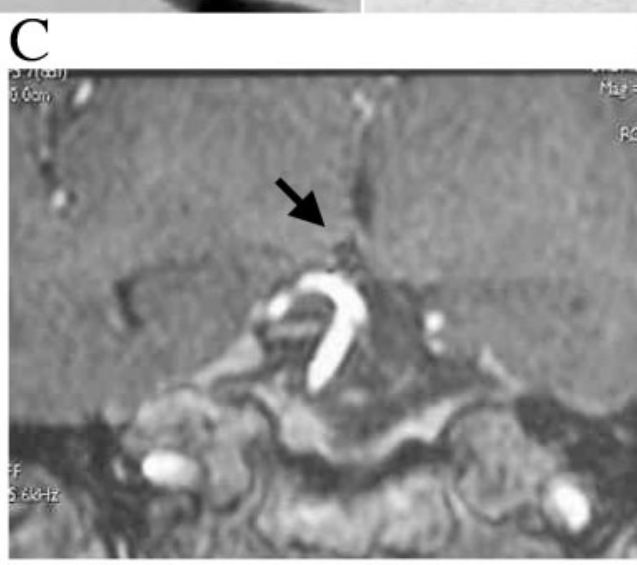

$\mathrm{E}$
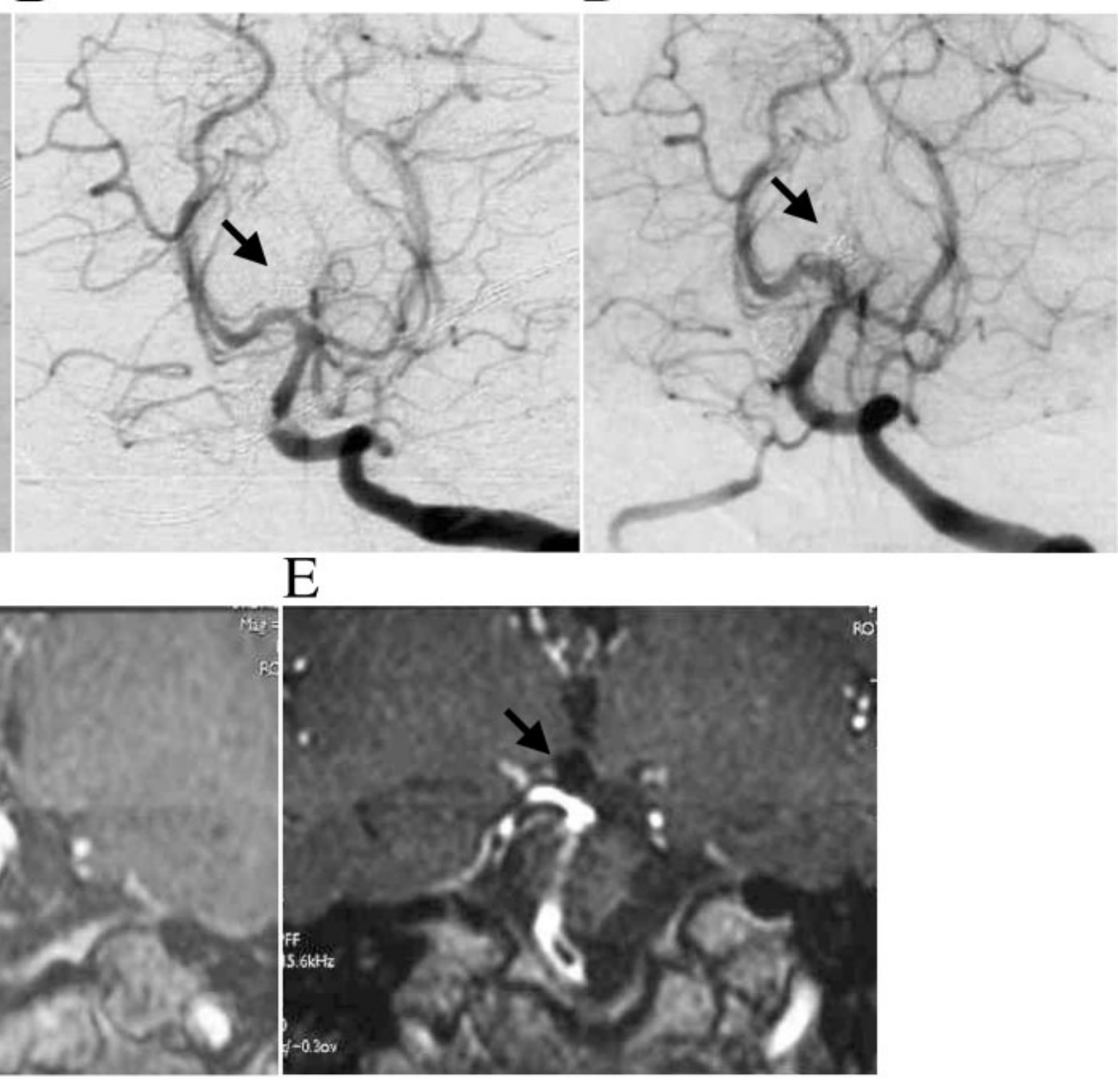

Figure 3: Case 1. Ruptured right posterior cerebral artery aneurysm successfully treated with coiling. (A) Digital subtraction angiography (DSA) on admission showed right posterior cerebral artery aneurysm; (B) post-procedural control angiogram and (C) 3D TOF MRA demonstrated complete occlusion of the aneurysm. (D) DSA 12 months later showed that occlusion remained unchanged. (E) Three-dimensional TOF MRA 4 years later demonstrated complete occlusion. Arrows indicate embolized aneurysm 


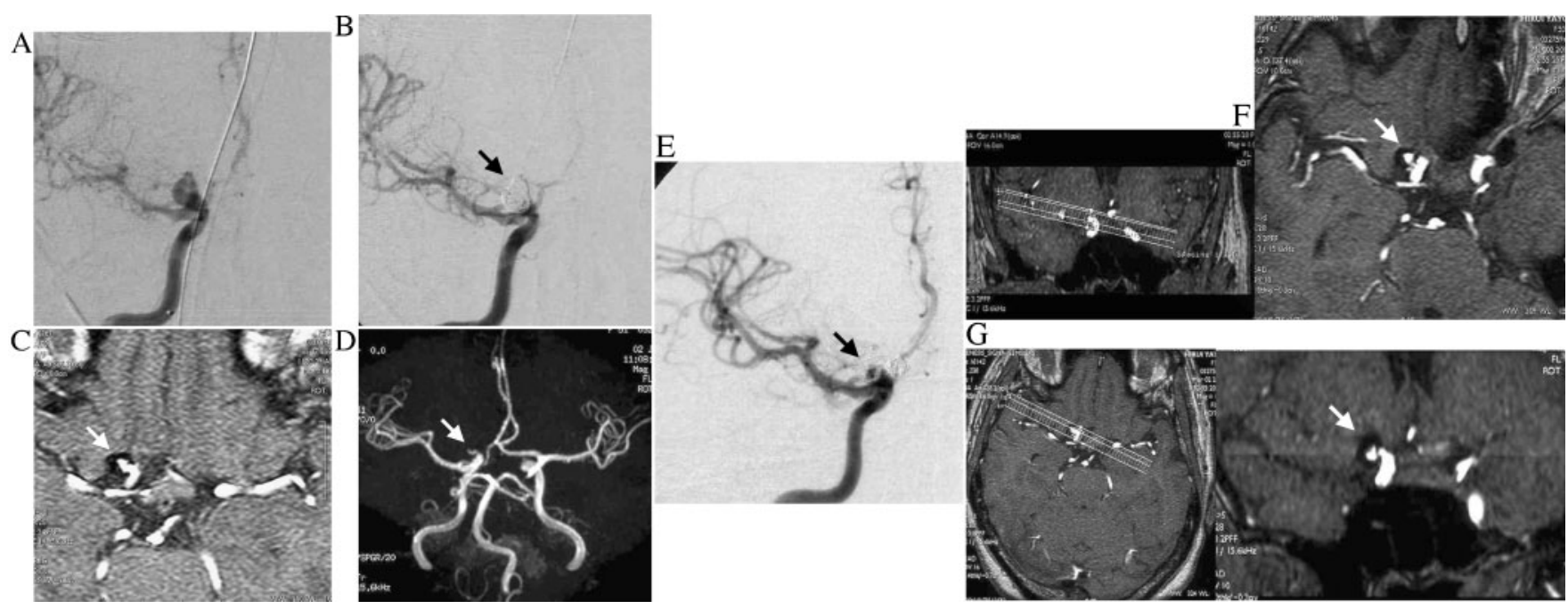

Figure 4: Case 2. (A) DSA on admission showed right internal carotid artery bifurcation aneurysm. (B) Post-procedural control angiogram showed minor filling of aneurysm. (C) An MRA MPR image showed significant filling of aneurysm, classified as central type. (D) Although an MRA MIP image also showed residual filling, resolution was not sufficient. (E) DSA 12 months later showed minor recurrence of aneurysm. Axial view $(\mathbf{F})$ and coronal view $(\mathbf{G})$ of 3D TOF MRA showed that residual filling remained stable. Arrows indicate embolized aneurysm

A

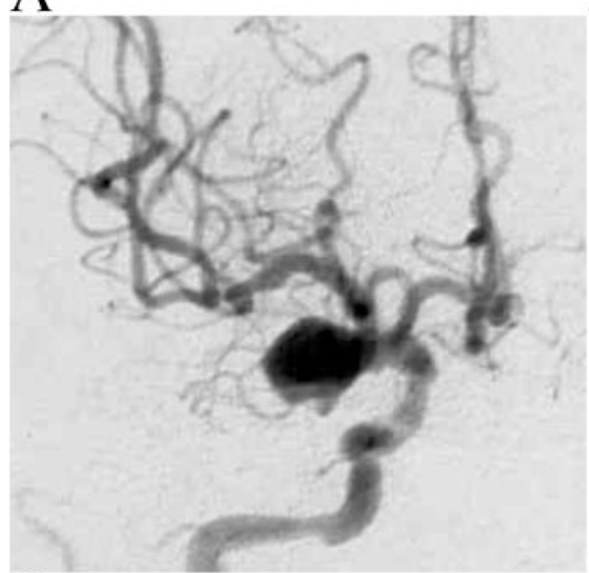

$\mathrm{C}$

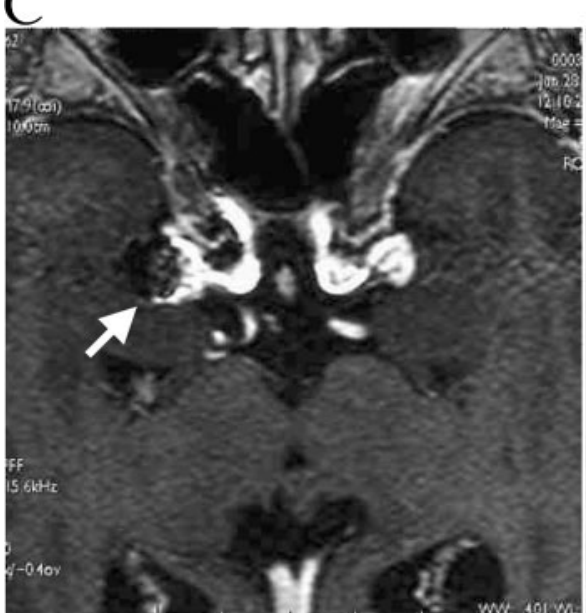

B

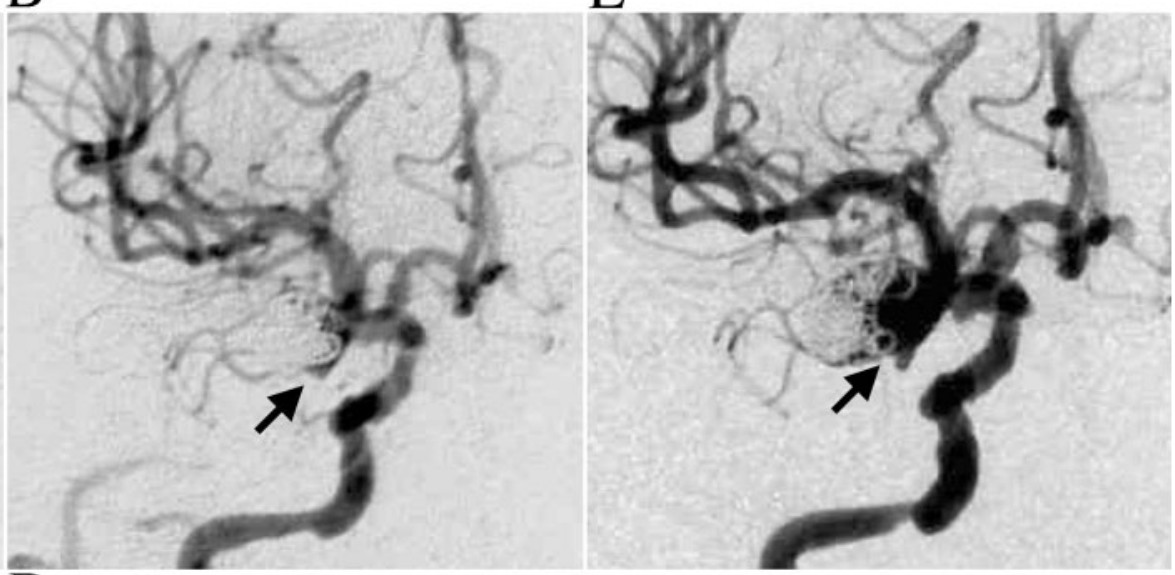

D

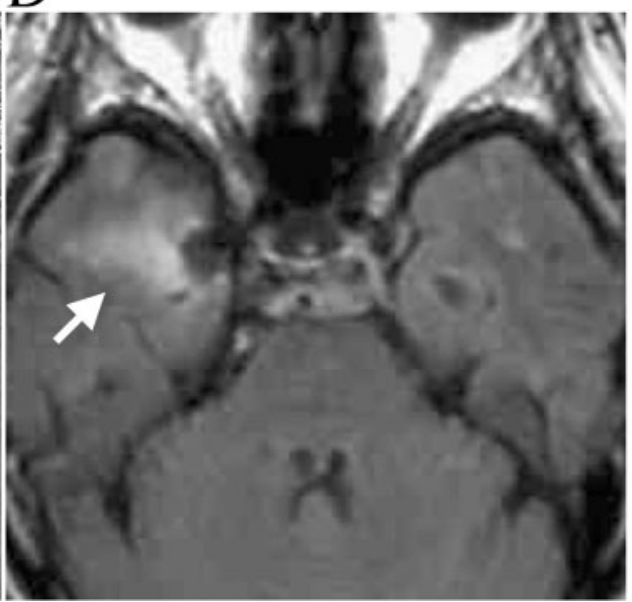

Figure 5: Case 3. (A) DSA on admission showed right internal carotid artery aneurysm. (B) Post-procedural control angiogram showed marginal filling at the neck. (C) Three-dimensional TOF MRA showed significant filling of the aneurysm, classified as residual dome marginal type. (D) MRI (fluid attenuation inversion recovery image) showed perianeurysmal edema. (E) Recurrence identified with DSA 18 months later. Arrows indicate embolized aneurysm 
A

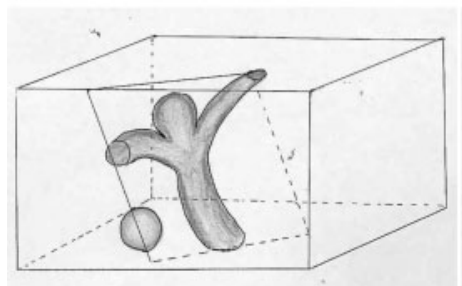

B

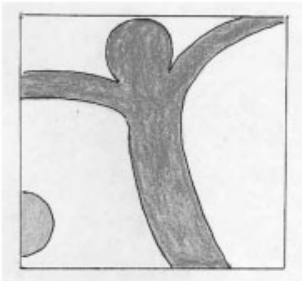

C

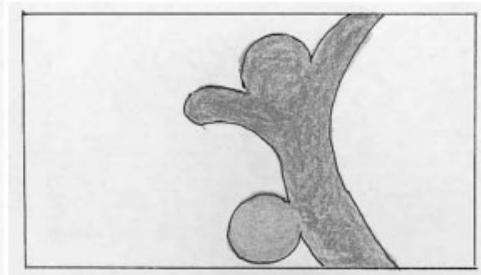

D

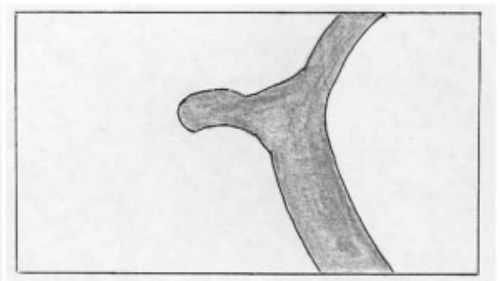

Figure 6: Schematic illustration of MRA. (A) Original data of 3D TOF MRA. (B) MPR image. This image shows best angle for the neck of the aneurysm in detail. (C, D) MIP image suitable for recognition of location of aneurysm. (C) However, this image may induce overlap of other hyperintensity region such as orbital fat. (D) A slow-flow part-like turbulent flow inside the aneurysm may not appear in MIP image

classified as a residual dome. An MPR image of 3D TOF MRA revealed a marginal-type filling. Furthermore, perianeurysmal edema was identified with MRI, and the aneurysmal wall was enhanced with a contrast medium. The aneurysm recurred 18 months later and was retreated with coil embolization.

\section{DISCUSSION}

Endovascular coil embolization has revolutionized the treatment of intracranial aneurysms. In a selected group of patients, endovascular and neurosurgical clip application are judged to be equivalent therapeutic options $^{1,2}$. However, growth of the aneurysm after coiling and subsequent rebleeding have been reported ${ }^{8}$. The critical issues are the stability of the occlusion after treatment with detachable coils and the efficacy in providing protection against growth or regrowth of the aneurysm $^{10,11}$. DSA is used to follow-up intracranial aneurysms treated with detachable coils to identify recurrence and the necessity for any additional treatment. However, DSA is invasive and involves a small risk of neurological complications ${ }^{12}$. For these reasons, a noninvasive technique such as MRA would be preferable if it could provide excellent characterization of cerebral vasculature.

Technical advances in MRA have continued to improve the sensitivity of this imaging technique for detecting cerebral aneurysms. Thus, 3D TOF MRA provides good spatial resolution, and aneurysms as small as $2 \mathrm{~mm}$ can now be detected ${ }^{13}$. Dealing with the 3D data, MPR and MIP images are available. An MPR image is the best position to visualize the residual flow of the neck in detail (Figure 6). As shown in Figure 3, MPR images revealed residual flow inside the coiled aneurysm because an artifact due to coil placement was minimal on MRA. These small remnants may have been obscured by the overlying coil mash on the DSA projection. Farb et al. ${ }^{14}$ termed this phenomenon as the 'helmet' effect. Although an MIP image is superior to show the location of the aneurysm, the resolution is not sufficient to reveal minor residual flow ${ }^{15}$. The relatively long acquisition time and the presence of flow-related artifacts are the limiting factors of this $\operatorname{method}^{16}$. In addition, there may be high-signal artifacts from intraluminal thrombus or perianeurysmal hemorrhage, which can be mistaken for flow signal (Figure 6). Contrast enhancement allows the imaging of low-flow signals. Theoretically, this allows for a higher conspicuity of a residual aneurysm $7,14,17$.

Each aneurysm was assigned to three categories as suggested previously: complete occlusion, residual neck and residual dome ${ }^{7-9}$. Complete obliteration was demonstrated in $18.8 \%$ of the aneurysms (12/64). Because 3D TOF MRA is sensitive in visualizing residual filling, the ratio of complete occlusion might be relatively low in this research. In fact, the rate of complete occlusion is quite controversial (16-85\%) according to the examination modalities ${ }^{7-9,18-20}$. In addition, the high sensitivity of 3D TOF MRA enables us to further classify the fillings as a central or marginal type. Fifty-eight patients with 64 intracranial saccular aneurysm treated with detachable coil were clinically followed-up and studied with both DSA and 3D TOF MRA. In this long-term follow-up study, recurrences were found in 18 aneurysms $(28.1 \%)$ and nine major recurrences were retreated with coils or surgery. One patient $(1.7 \%)$ experienced a bleeding episode during the follow-up period. These results were consistent with previous reports, in which angiographic recurrences were observed in $33.6 \%$ of cases and an annual delayed rebleeding rate from 0.9 to $1.4 \%{ }^{9}$.

The correlation between recurrence and classification of the residual filling was evaluated. A strong correlation was found between the immediate post-treatment radiological classification and the likelihood of future adverse events such as aneurysm recurrence, retreatment or clinical deterioration. It has been reported that significant predictors of a recurrence included aneurysm size, treatment during acute phase rupture and incomplete initial occlusions ${ }^{8,9}$. In addition, we demonstrated that the type of residual filling strongly influence the outcome in this series. Unfortunately, we could not evaluate the relation between the coiling procedure and the immediate results, but we speculate that the causes of the residual filling are as follows: first, in the case of the central-type filling, the first coil was properly placed into the aneurysm covering including neck. However, the last coils were not inserted because of the presence of coils, resulting to a residual filling at the center (Figure 7A). Against that, the first coil did not cover the whole aneurysm in the case of the marginal-type filling. This may be seen in smaller caging coil for aneurysm size or a 3D coil, which does not always fit the shape of the aneurysm, resulting to a dog-eared remnant (Figure $7 B$ ). In the case of the central-type filling, the 
A

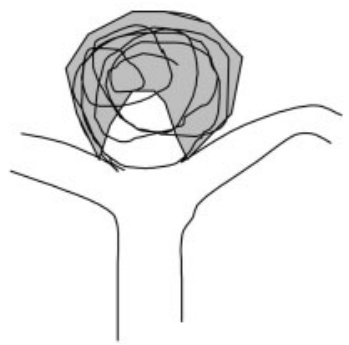

B

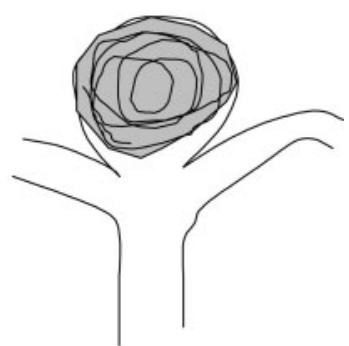

Figure 7: Schematic illustration of residual filling and coiling: (A) central-type filling and (B) marginal-type filling

wall of aneurysm was covered with coils and protected from blood flow. Therefore, this type of filling was relatively stable during follow-up. Against that, blood flow directly affects the wall of aneurysm in the marginal-type filling. This mechanical stress or local inflammation may be correlated with regrowth of the aneurysm.

We have so far reported three cases of perianeurysmal edema after coil embolization ${ }^{21}$. Five cases with perianeurysmal edema were identified totally, and all of them recurred in our series. This response may result from focal inflammation or neovascularization of the aneurysmal wall and be correlated with regrowth of the embolized aneurysm. Because these responses were observed before recurrence, these signs may be useful to predict recurrence of the lesion.

\section{CONCLUSION}

The three-dimensional TOF MRA was sensitive to visualize the residual filling of the embolized aneurysm. The recurrences were frequently seen in the marginaltype filling. Three-dimensional TOF MRA is a feasible and useful tool for follow-up of patients with intracranial aneurysms treated with coil placement.

\section{REFERENCES}

1 Molyneux AJ, Kerr RS, Yu LM, et al. International Subarachnoid Aneurysm Trial (ISAT) Collaborative Group. International Subarachnoid Aneurysm Trial (ISAT) of neurosurgical clipping versus endovascular coiling in 2143 patients with ruptured intracranial aneurysms: A randomised comparison of effects on survival, dependency, seizures, rebleeding, subgroups, and aneurysm occlusion. Lancet 2005; 366: 809-817

2 Molyneux AJ, Kerr R, Stratton I, et al. International Subarachnoid Aneurysm Trial (ISAT) Collaborative Group. International Subarachnoid Aneurysm Trial (ISAT) of neurosurgical clipping versus endovascular coiling in 2143 patients with ruptured intracranial aneurysms: A randomized trial. Lancet 2002; 360: 1267-1274

3 Byrne JV, Sohn MJ, Molyneux AJ, et al. Five-year experience in using coil embolization for ruptured intracranial aneurysms: Outcomes and incidence of late rebleeding. J Neurosurg 1999; 90: $656-663$
4 Hartman J, Nguyen T, Larsen D, et al. MR artifacts, heat production, and ferromagnetism of Guglielmi detachable coils. AJNR Am J Neuroradiol 1997; 18: 497-501

5 Kahara VJ, Seppanen SK, Ryymin PS, et al. MR angiography with three-dimensional time-of-flight and targeted maximum-intensityprojection reconstructions in the follow-up of intracranial aneurysms embolized with Guglielmi detachable coils. AJNR Am J Neuroradiol 1999; 20: 1470-1475

6 Yamada N, Hayashi K, Murao K, et al. Time-of-flight MR angiography targeted to coiled intracranial aneurysms is more sensitive to residual flow than is digital subtraction angiography. AJNR Am J Neuroradiol 2004; 25: 1154-1157

7 Gauvrit JY, Leclerc X, Caron S, et al. Intracranial aneurysms treated with Guglielmi detachable coils: Imaging follow-up with contrastenhanced MR angiography. Stroke 2006; 37: 1033-1037

8 Murayama Y, Nien YL, Duckwiler G, et al. Guglielmi detachable coil embolization of cerebral aneurysms: 11 years' experience. J Neurosurg 2003; 98: 959-966

9 Raymond J, Guilbert F, Weill A, et al. Long-term angiographic recurrences after selective endovascular treatment of aneurysms with detachable coils. Stroke 2003; 34: 1398-1403

10 Gallas S, Pasco A, Cottier JP, et al. A multicenter study of 705 ruptured intracranial aneurysms treated with Guglielmi detachable coils. AJNR Am J Neuroradiol 2005; 26: 1723-1731

11 Kang HS, Han MH, Kwon BJ, et al. Repeat endovascular treatment in post-embolization recurrent intracranial aneurysms. Neurosurgery 2006; 58: 60-70

12 Willinsky RA, Taylor SM, TerBrugge K, et al. Neurologic complications of cerebral angiography: Prospective analysis of 2,899 procedures and review of the literature. Radiology 2003; 227: $522-528$

13 Majoie CB, Sprengers ME, van Rooij WJ, et al. MR angiography at $3 \mathrm{~T}$ versus digital subtraction angiography in the follow-up of intracranial aneurysms treated with detachable coils. AJNR Am J Neuroradiol 2005; 26: 1349-1356

14 Farb RI, Nag S, Scott JN, et al. Surveillance of intracranial aneurysms treated with detachable coils: A comparison of MRA techniques. Neuroradiology 2005; 47: 507-515

15 Anzalone N, Righi C, Simionato F, et al. Three-dimensional timeof-flight MR angiography in the evaluation of intracranial aneurysms treated with Guglielmi detachable coils. AJNR Am J Neuroradiol 2000; 21: 746-752

16 Cottier JP, Bleuzen-Couthon A, Gallas S, et al. Follow-up of intracranial aneurysms treated with detachable coils: Comparison of plain radiographs, 3D time-of-flight MRA and digital subtraction angiography. Neuroradiology 2003; 45: 818-824

17 Leclerc X, Navez JF, Gauvrit JY, et al. Aneurysms of the anterior communicating artery treated with Guglielmi detachable coils: Follow-up with contrast-enhanced MR angiography. AJNR Am J Neuroradiol 2002; 23: 1121-1127

18 Cognard C, Weill A, Castaings L, et al. Intracranial berry aneurysms: Angiographic and clinical results after endovascular treatment. Radiology 1998; 206: 499-510

19 Grunwald IQ, Papanagiotou P, Struffert T, et al. Recanalization after endovascular treatment of intracerebral aneurysms. Neuroradiology 2007; 49: 41-47

20 Kole MK, Pelz DM, Kalapos P, et al. Endovascular coil embolization of intracranial aneurysms: Important factors related to rates and outcomes of incomplete occlusion. J Neurosurg 2005; 102: 607-615

21 Horie N, Kitagawa N, Morikawa M, et al. Progressive perianeurysmal edema induced after endovascular coil embolization report of three cases and review of the literature. J Neurosurg 2007; 106: 916-920 